J. Lake Sci.(湖泊科学), 2019, 31(1):243-255

DOI 10. 18307/2019. 0123

(c) 2019 by Journal of Lake Sciences

\title{
藏南羊卓雍错湖面大气湍流特征观测分析
}

\author{
沈鹏珂 $^{1,2}$, 张雪芹 $^{1 \text { ** }}$ \\ (1: 中国科学院地理科学与资源研究所,中国科学院陆地表层格局与模拟重点实验室, 北京 100101) \\ (2: 中国科学院大学,北京 100049)
}

\begin{abstract}
摘 要: 湍流运动是大气边界层的本质特征, 是地表与大气之间能量和物质交换的主要方式. 本文利用 2016 和 2017 年 4-10 月藏南羊卓雍错湖泊浴动观测资料, 分析了湖面大气湍流方差和湍流特征量的统计和变化特征. 结果表明: (1) 不 稳定层结下, 三维风速分量和超声虚温、水汽密度、 $\mathrm{CO}_{2}$ 密度的无量纲标准差随稳定度变化符合 Monin-Obukhov 相似理论 的 “ $1 / 3$ ” 或 “ $-1 / 3$ ” 次幂律, 垂直风速的拟合效果最好; 稳定层结下, 除 $\mathrm{CO}_{2}$ 密度无量纲标准差与稳定度无明显关系外, 其 他量基本上满足相似性规律; 中性条件下, 以上物理量的无量纲标准差分别趋近常数: 3.57、3.93、0.77、20.91、6.35 和 11.96. (2) 水平方向平均湍流强度 (0.60 和 0.58) 大于垂直方向 $(0.13)$, 三维方向湍流强度与平均风速的变化呈显著负相 关, 相关系数分别为 $-0.39 、-0.42$ 和-0.34. (3) 湖面湍流动能随风速呈线性增长, 增长率达 $0.45 \mathrm{~m} / \mathrm{s}$; 近中性层结时湍流 动能最大, 层结越稳定或不稳定湍流动能均减小. (4) 湖泊下午到傍晚动量输送较强, 13:00-22:30 时间段平均动量通量 达 $0.091 \mathrm{~kg} /\left(\mathrm{m} \cdot \mathrm{s}^{2}\right)$; 热量输送以潜热为主, 潜热通量日平均值 $\left(77.3 \mathrm{~W} / \mathrm{m}^{2}\right)$ 是感热通量 $\left(14.6 \mathrm{~W} / \mathrm{m}^{2}\right)$ 的 5.3 倍, 感热和潜 热通量日变化峰值分别出现在 $5: 30\left(22.4 \mathrm{~W} / \mathrm{m}^{2}\right)$ 和 $16: 00\left(106.6 \mathrm{~W} / \mathrm{m}^{2}\right)$.
\end{abstract}

关键词: 相似理论; 湍流通量; 湍流动能; 浴动相关法; 羊卓雍错

\section{Observation of atmospheric turbulence characteristics over the Yamzhog Yumco, south Tibet}

\author{
SHEN Pengke ${ }^{1,2} \&$ ZHANG Xueqin ${ }^{1 * *}$ \\ (1: Key Laboratory of Land Surface Pattern and Simulation, Institute of Geographic Sciences and Natural Resources Research, \\ Chinese Academy of Sciences, Beijing 100101, P.R.China) \\ (2: University of Chinese Academy of Sciences, Beijing 100049, P.R.China)
}

\begin{abstract}
Turbulent motion is the essential characteristic of atmospheric boundary layer, as well as the main pathway of atmospheric energy and mass transfer. The atmospheric turbulent variance and characteristic quantities are investigated over the Yamzhog Yumco based on eddy covariance data during April-October 2016 and 2017. The main results are summarized as follows. (1) The dimensionless standard deviations of velocity components, sonic temperature, vapor density, and $\mathrm{CO}_{2}$ density varying with stability follow the law of “ $1 / 3$ ” or “-1/3" fractional power of Monin-Obukhov Similarity Theory in unstable stratification, and the fitting effect of vertical speed is optimal. The dimensionless standard deviations of all physical quantities except $\mathrm{CO}_{2}$ density, versus stability are also in agreement with the fractional power law in stable stratification. Under the near neutral stratification, these dimensionless standard deviations reach constants : 3.57, 3.93, 0.77, 20.91, 6.35 and 11.96, respectively. (2) The horizontal turbulent intensities (on average, 0.60 and 0.58 ) are larger than vertical turbulent intensity $(0.13$ ), and the variations of three-dimensional turbulent intensities with average wind speed show significant negative correlation with the coefficients of $-0.39,-0.42$ and -0.34 , respectively. (3) Turbulent kinetic energy increases with wind speed with the rate of $0.45 \mathrm{~m} / \mathrm{s}$, and it displays stronger in near neutral stratification, while decreases when the atmosphere becomes stable or unstable. (4) Momentum flux is large during 13:00$22: 30$ with the average of $0.091 \mathrm{~kg} /\left(\mathrm{m} \cdot \mathrm{s}^{2}\right)$. Latent heat flux, 5.3 times higher than sensible heat flux with daily average value $\left(14.6 \mathrm{~W} / \mathrm{m}^{2}\right)$, plays a leading role in heat transportation. Sensible and latent heat flux reach maximum value at 5:30 (22.4 W/
\end{abstract}

* 国家自然科学基金项目 (41471064) 资助. 2018-04-15 收稿; 2018-05-16 收修改稿. 沈鹏珂 (1992 ), 男,硕士研 究生;E-mail: shenpk.15s@igsnrr.ac.cn.

** 通信作者;E-mail:zhangxq@ igsnrr.ac.cn. 
$\left.\mathrm{m}^{2}\right)$ and 16:00 $\left(106.6 \mathrm{~W} / \mathrm{m}^{2}\right)$, respectively.

Keywords: Similarity theory; turbulent fluxes; turbulent kinetic energy; eddy-covariance measurements; Yamzhog Yumco

高寒湖泊是青藏高原地表系统的重要组成部分, 因受人类影响小、多处于自然状态, 能更加敏感、真实 地反映自然气候变化 ${ }^{[1]}$. 与周边陆地相比, 湖泊具有反照率低、粗䊅度小和比热容大等特点, 导致其储存更 多太阳辐射、对气温变化起缓冲作用 ${ }^{[2]}$, 并影响区域气候 ${ }^{[3]}$. 如湖泊和陆地热力差异所激发的局地热力环流 影响着流域内的大气环境以及污染物的输送 ${ }^{[4]}$, 湖泊提供充分水汽、增强下游降水等 ${ }^{[5-6]}$. 上述影响主要通 过湖泊和大气间的相互作用实现, 而大气湍流活动则是湖一气相互作用的主要方式. 因此, 对高寒湖泊大气 湍流的直接观测及分析具有重要意义.

浴动相关法是直接测量地表湍流通量的方法, 通过高频 $\left(\sim 10 \mathrm{~Hz}\right.$ ) 观测垂直风速和研究变量 ( 如 $\mathrm{CO}_{2}$ 和 $\mathrm{H}_{2} \mathrm{O}$ ), 计算两者脉动协方差得到通量 ${ }^{[6]}$. 相比其他方法, 浴动相关法既不是建立在经验关系基础之上, 亦不 是从其他气象参量推论出来, 在计算过程中几乎不存在任何假设. 国内已报道的内陆湖泊浴动相关观测主 要集中在太湖 ${ }^{[7-9]} 、$ 、鄱阳湖 ${ }^{[10-11]} 、$ 纳木错 ${ }^{[12-13]} 、$ 、洱海 ${ }^{[14]}$ 和巴丹吉林沙漠湖泊 ${ }^{[15]}$. 高时间分辨率的通量直接观 测丰富了对湖一气相互作用过程的认识, 如影响感热和潜热通量日变化的主控因素分别是湖一气温差和饱 和水汽压差 ${ }^{[14,16]}$; 湖面感热和潜热通量日变化峰值分别出现在清晨和下午, 而非太阳辐射最强的正 午 $^{[8,11,16]}$, 主要得益于水体热储量释放 ${ }^{[17]}$; 在小时尺度上, 风速主导湍流热交换变化 ${ }^{[13]}$, 影响蒸发的主要因 子为风速、饱和水汽压差的乘积, 而非净辐射 ${ }^{[17-18]}$.

湍流运动是大气边界层的本质特征 ${ }^{[19]}$, 尤其在大气边界层底层一一近地层, 科氏力与气压梯度力的作 用相对湍流切应力可忽略, 大气结构主要依赖垂直湍流输送 ${ }^{[20]}$. 近地层湍流研究中, 验证 Monin-Obukhov 相 似理论 ${ }^{[21]}$ 的适用性是认识地-气相互作用的重要前提. 已有研究证实 ${ }^{[22-24]}$, 高原地区近地层风速等变量满 足类似平原地区所得 “ $1 / 3$ ” 和“ $-1 / 3$ ” 次幂律, 垂直风速的相似性优于水平风速 ${ }^{[25]}$; 温度、湿度仅在不稳定层 结下服从幂律, 稳定层结下则关系消失 ${ }^{[26-27]}$. 尽管 Monin-Obukhov 相似理论是普适的, 不同地区或下垫面湍 流发展的强弱规律符合相似性的程度以及普适函数系数取值均存在较大差异性. 并且, 利用所获相似关系 和拟合系数, 可进一步通过 “通量一方差法” 估算湍流通量、填补因风速观测无效而引起的通量数据空

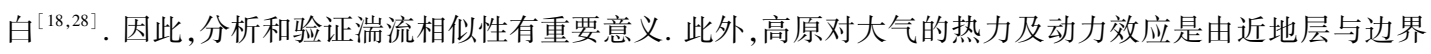
层逐渐影响到自由大气, 故对高原边界层微气象特征的分析研究尤为重要 ${ }^{[25]}$. 已有关于湍流宏观统计特征 量的研究多集中于湍流强度、湍流动能的数值分布及其与风速、稳定度的关系 ${ }^{[24,29]}$, 以及动量、热通量变化 规律和成因等方面 ${ }^{[14,30-31]}$. 基于浴动相关观测系统, 已在高原多地区陆地下垫面开展近地层湍流特征研究, 而湖泊下垫面报道相对较少, 仅限于青海湖 ${ }^{[32]}$ 、色林错 ${ }^{[31]}$ 、鄂陵湖 ${ }^{[33]}$ 及纳木错 ${ }^{[12-13]}$ 等, 对高原南部湖泊的 近地层湍流特征研究更是未涉及, 亟待扩展和加强高原湖泊大气湍流的观测分析和深人探究.

羊卓雍错位于青藏高原南部, 是雅鲁藏布江南岸和喜马拉雅山北麓最大的内陆湖泊. 最近 10 年该湖泊 面积萎缩 ${ }^{[34]}$ 、水位急剧下降, 大片湖床出露, 已威胁到湖区及周围农牧民生产生活和生态环境安全, 引起当 地政府高度重视. 目前亟需对该湖泊水热循环及气候环境效应方面开展综合研究. 然而自然环境严酷导致 的观测数据圄乏成为羊卓雍错湖泊研究的瓶颈. 2016 年 3 月, 本研究组在该湖泊安装浴动相关系统开展长 期定点观测. 本文旨在利用 2016 和 2017 年 4-10 月所获浴动观测资料, 分析湖面大气湍流方差统计特征, 验证边界层相似理论在该湖泊的适用性, 分析湍流强度、湍流动能和湍流通量的统计和变化规律, 以期增进 对高原湖泊边界层湍流交换规律的认识, 为改进高原湖泊气候模型参数化方案和进一步研究高寒湖泊水热 循环过程提供基础数据和理论支撑.

\section{1 资料与方法}

\section{1 观测仪器和数据预处理}

羊卓雍错流域海拔 $4400 \mathrm{~m}$ 以上, 属藏南山地灌丛草原半干旱气候, 多年平均气温和降水量分别为 2.9 ${ }^{\circ} \mathrm{C}$ 和 $365.7 \mathrm{~mm}$ ( 根据浪卡子气象站 1961-2014 年记录). EC3000 开路浴动相关系统架设在流域白地水文站 旁边的湖岸浅水区 $\left(29^{\circ} 07^{\prime} 28^{\prime \prime} \mathrm{N}, 90^{\circ} 26^{\prime} 27^{\prime \prime} \mathrm{E}\right.$ ), 海拔 $4420.6 \mathrm{~m}$. 主体仪器是 IRGASON 浴动相关 (Campbell Sci- 
entific, USA), 2016 和 2017 年安装朝向角分别为正北顺时针 $170^{\circ}$ 和 $178^{\circ}$,距水面 $2.1 \mathrm{~m}$ (安装时), 主要采集 数据有三维风速、超声虚温、气压、水汽密度和 $\mathrm{CO}_{2}$ 密度, 采集频率为 $10 \mathrm{~Hz}$. 选配仪器包括空气温湿度传感 器( HMP155A, Campbell Scientific, USA)、四分量净辐射传感器 ( CNR4, Kipp \& Zonen, Netherlands)、红外温度 传感器( SI-111, Apogee, USA) 和雨量筒( TE525MM, Campbell Scientific, USA), 测量气温、相对湿度、辐射四分 量、湖面温度 (由红外温度传感器直接观测) 和降水量等指标, 由数据采集器 (CR3000-NB-XT, Campbell Scientific, USA) 处理成 $30 \mathrm{~min}$ 平均值并储存. 数据记录时间为北京时间, 根据经度差, 分析要素日变化时减去 2 $\mathrm{h}$ 换算为本地时间.

资料收集跨度为 2016 和 2017 年 4-10 月,使用美国 LI-COR 公司研发的 EddyPro 6.1 软件( Eddy covariance processing software, http://www.licor.com/eddypro) 进行处理,平均时间取 $30 \mathrm{~min}$. 主要处理包括: 二次坐 标旋转修正、WPL 密度修正、超声虚温修正及高、低通滤波修正等 ${ }^{[35]}$. 涡动相关法的应用主要基于 3 个假设 (稳态、地形平坦、下垫面性质均匀一致 $)^{[36]}$, 而实际观测条件往往无法满足. 因此, 观测数据包含有湍流不 平稳性以及偏离湍流特征的无效数据, 需要对其进行质量控制. 本文采取 Foken 等提出的质量控制方法, 包 括湍流平稳性检验、湍流发展充分性检验 ${ }^{[36]}$. 当 EddyPro 软件输出 $30 \mathrm{~min}$ 动量、感热和潜热通量的同时, 伴 随输出相应总质量级标记: “ 0 ”代表高质量通量,可用于基础研究, 如参数化方案的发展; “ 1 ”代表中等质量 数据, 可用于长期观测资料处理; “ 2 ”代表低质量数据, 本文予以剔除处理.

\section{2 湖面源区划分}

涡动相关仪器为定点观测, 能反映周围整个观测区域状况. 通量贡献区是指对观测点湍流交换有贡献 的有效源 (汇) 区域, 即对观测点通量大小产生主要影响的表面区域 ${ }^{[37]}$. 本文使用 Kljun 通量源区模型 ${ }^{[38]}$ 进 行源区分析, 该模型是基于尺度 (量纲) 分析衍生的新颖算法, 主要用来计算通量足迹的侧风积分函数. 根据 2016 年 3 月 26 日 Landsat 8 遥感影像, 结合由 EddyPro 输出的累积 90\% 通量贡献率区域至观测点的距离, 本 文取观测点 $70^{\circ} \sim 180^{\circ}$ 、观测点向东 $240 \mathrm{~m}$ 处 $90^{\circ} \sim 130^{\circ}$ 范围代表湖泊通量源 (图 1 ). 经统计, 表征湖泊通量 源的频次为 7471 , 占观测时段 (2016 年和 2017 年 4-10 月) 的 $39.4 \%$.

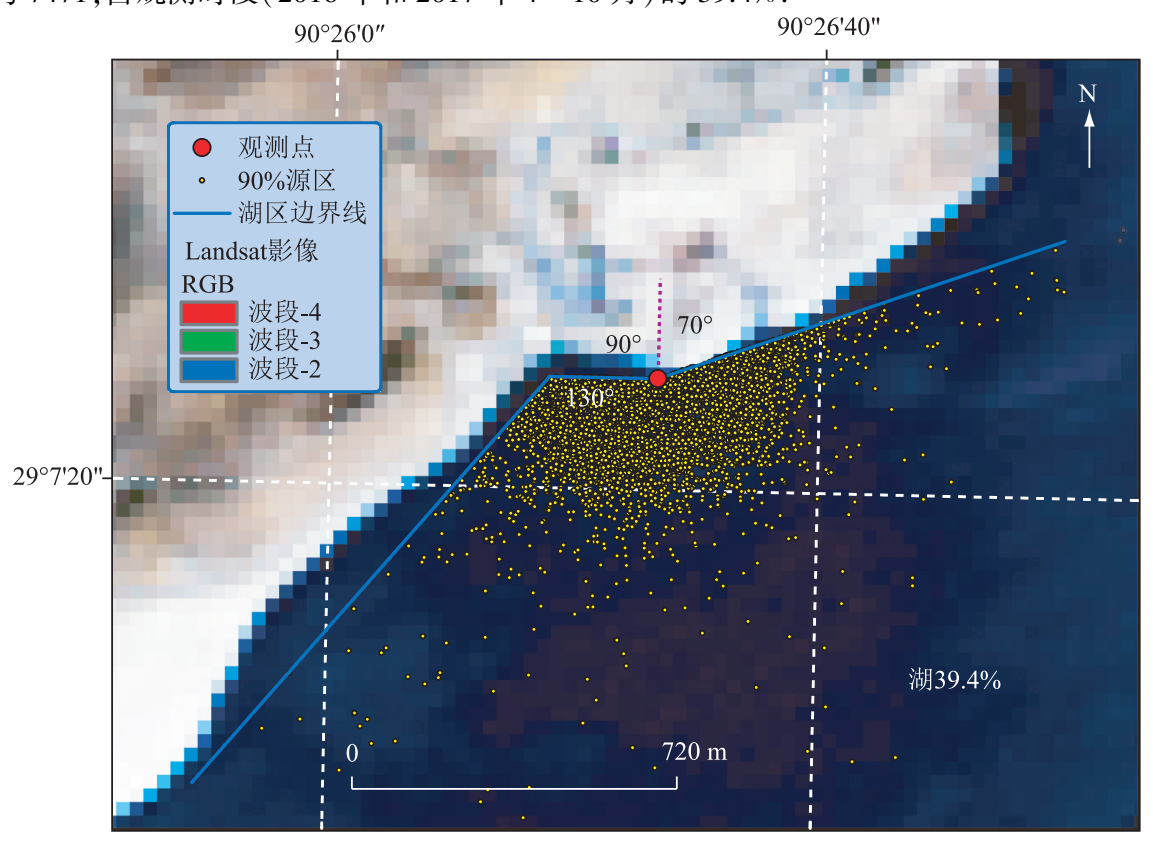

图 12016 和 2017 年 4-10 月羊卓雍错湖面通量源区分布(黄色点代表累积 $90 \%$

通量贡献的远端点, 背景为 Landsat 8 卫星遥感影像波段 4、3、2 组合, 时间为 2016 年 3 月 26 日)

Fig. 1 Distribution of flux source area in Yamzhog Yumco during April-October 2016 and 2017

( The yellow points denote the distal points of the along-wind distance providing $90 \%$ (cumulative) contribution to turbulent fluxes; The background is Landsat 8 satellite image (bands 4, 3 and 2) on March 26th, 2016) 


\section{3 主要湍流特征量计算}

1.3.1 湍流强度 湍流强度 $(I)$ 反映风的脉动强度, 三维风分量的湍流强度分别表示为:

$$
I_{\mathrm{u}}=\sigma_{\mathrm{u}} / U, I_{\mathrm{v}}=\sigma_{\mathrm{v}} / U, I_{\mathrm{w}}=\sigma_{\mathrm{w}} / U
$$

式中, $U=\sqrt{u^{2}+v^{2}+w^{2}}$, 为平均风速 $(\mathrm{m} / \mathrm{s}) ; \sigma_{u} 、 \sigma_{v} 、 \sigma_{\mathrm{w}}$ 分别为三维风速瞬时量 $(u 、 v 、 w)$ 的标准差 $(\mathrm{m} / \mathrm{s})$. 1.3.2 湍流动能 湍流动能 $\left(T K E, \mathrm{~m}^{2} / \mathrm{s}^{2}\right)$ 是湍流强度的量度, 直接关系边界层大气动量、热量及水汽等属性 的输送, 定义为 ${ }^{[39]}$ :

$$
T K E=\left(\sigma_{u}^{2}+\sigma_{v}^{2}+\sigma_{w}^{2}\right) / 2
$$

式中, $\sigma_{u}^{2} 、 \sigma_{v}^{2} 、 \sigma_{w}^{2}$ 是三维风速瞬时量的方差 $\left(\mathrm{m}^{2} / \mathrm{s}^{2}\right)$.

1.3.3 湍流通量 浴动相关法是根据微气象学原理, 计算垂直风速脉动量与有关物理要素脉动量的协方差获 取湍流通量. EddyPro 计算动量通量 $\left(\tau, \mathrm{kg} /\left(\mathrm{m} \cdot \mathrm{s}^{2}\right)\right)$ 、感热通量 $\left(H, \mathrm{~W} / \mathrm{m}^{2}\right)$ 和潜热通量 $\left(\lambda E, \mathrm{~W} / \mathrm{m}^{2}\right)$ 的方 法为 ${ }^{[35]}$ :

$$
\left\{\begin{array}{c}
\tau=\rho_{\mathrm{a}} u_{*}^{2} \\
H=\rho_{\mathrm{a}} C_{\mathrm{P}} \overline{w^{\prime} T_{\mathrm{s}}{ }^{\prime}} \\
\lambda E=\lambda \overline{w^{\prime} d_{\mathrm{H}_{2} \mathrm{O}}^{\prime}}
\end{array}\right.
$$

式中, $\left.u_{*}=\left(\overline{u^{\prime} \cdot w^{\prime}}{ }^{2}+\overline{v^{\prime} \cdot w^{\prime}}\right)^{2}\right)^{1 / 4}$, 为摩擦速度 $(\mathrm{m} / \mathrm{s}) ; w^{\prime} 、 T_{\mathrm{s}}^{\prime} 、 d_{\mathrm{H}_{2}}^{\prime}$ 分别为垂直风速 $(\mathrm{m} / \mathrm{s})$ 、超声虚温 $(\mathrm{K})$ 和 水汽密度 $\left(\mathrm{mg} / \mathrm{m}^{3}\right)$ 的脉动量. 湿空气密度 $\left(\rho_{\mathrm{a}}, \mathrm{kg} / \mathrm{m}^{3}\right)$ 、湿空气定压比热容 $\left(C_{\mathrm{P}}, \mathrm{J} /(\mathrm{kg} \cdot \mathrm{K})\right)$ 和蒸发潜热 $(\lambda, \mathrm{J} /$ $\mathrm{kg})$ 的计算详见手册 ${ }^{[35]}$.

\section{4 方差相似性验证}

在近地层相似理论中, 近地层湍流发展的强弱规律符合 Monin-Obukhov 相似性理论. 近地层风速分量 $(u 、 v 、 w)$ 、超声虚温 $\left(T_{\mathrm{s}}\right)$ 、水汽密度 $\left(d_{\mathrm{H}_{2} \mathrm{O}}\right)$ 和 $\mathrm{CO}_{2}$ 密度 $\left(d_{\mathrm{CO}_{2}}\right)$ 的标准差被其特征尺度参数标准化 (即无量纲 化) 后, 可以用大气静力稳定度 $(\zeta)$ 的普适函数表示. 多数研究证实, $u 、 v 、 w$ 的无量纲化标准差 $\left(\sigma_{x} /\left|X_{*}\right|\right)$ 随 $\zeta$ 的变化符合 Panofsky 等 ${ }^{[40]}$ 给出的 “ $1 / 3$ ” 次幂规律; 对于 $T_{\mathrm{s}} 、 d_{\mathrm{H}_{2} 0} 、 d_{\mathrm{CO}_{2}}$, 本文通过数据拟合, 发现其函数式 较符合 Tillman ${ }^{[41]}$ 给出的关系式; 另外, 无量纲湍流动能 $\left(T K E / u_{*}^{2}\right)$ 与 $\zeta$ 也存在与风速分量类似的表达. 各式 表达如下:

$$
\left\{\begin{array}{l}
\sigma_{x 1} /\left|X_{*}\right|=C_{1}\left(1 \pm C_{2} \zeta\right)^{\frac{1}{3}} \\
\sigma_{x 2} /\left|X_{*}\right|=C_{3}\left(C_{4} \pm \zeta\right)^{-\frac{1}{3}} \\
\text { TKE } / u_{*}^{2}=C_{5}\left(1 \pm C_{6} \zeta\right)^{\frac{1}{3}}
\end{array}\right.
$$

式中, $\sigma_{x 1} 、 \sigma_{x 2}$ 分别为参数 $x_{1}=(u 、 v 、 w) 、 x_{2}=\left(T_{\mathrm{s}} 、 d_{\mathrm{H}_{2} 0} 、 d_{\mathrm{CO}_{2}}\right)$ 的标准差, $X_{*}$ 为各参数对应的特征尺度 $\left(u_{*}\right.$ 、 $\left.t_{*} 、 h_{*}, c_{*}\right): t_{*}=-\overline{w^{\prime} T_{\mathrm{s}}{ }^{\prime}} / u_{*}, h_{*}=-\overline{w^{\prime} d_{\mathrm{H}_{2} 0}^{\prime}} / u_{*} 、 c_{*}=-\overline{w^{\prime} d^{\prime} \mathrm{CO}_{2}} / u_{*}$. 大气静力稳定度表征空气受垂直方 向扰动后, 大气层结使该空气团具有返回或远离原来平衡位置的趋势和程度. 本文取 $\zeta>0$ 表示稳定大气, $\zeta<0$ 表示不稳定大气. 以 Monin-Obukhov 相似理论和量纲分析为基础, $\zeta$ 的计算方法为 ${ }^{[39]}$ :

$$
\zeta=\frac{z-d}{L}
$$

式中, $z$ 为观测高度, 取 $2.1 \mathrm{~m}$, 忽略 4-10 月间湖水位的变化; $d$ 为零平面位移高度, 取 $0 \mathrm{~m} ; L$ 是 Obukhov 长 度, 是表征稳定度的一个特征参数, 计算式为 ${ }^{[35]}$ :

$$
L=-\frac{T_{\mathrm{p}} u_{*}^{3}}{\kappa g \overline{w^{\prime} T_{\mathrm{s}}{ }^{\prime}}}
$$

其中, $\kappa=0.41, g=9.81 \mathrm{~m} / \mathrm{s}^{2}, T_{\mathrm{p}}=T_{\mathrm{a}}\left(10^{5} / P_{\mathrm{a}}\right)^{0.286}, T_{\mathrm{a}}$ 和 $P_{\mathrm{a}}$ 分别为环境温度 $(\mathrm{K})$ 和压强 $(\mathrm{Pa})$.

本文选取 1stOpt 软件平台 (7D-Soft High Technology Inc., http://1 stopt. software.informer.com/) “麦夸特法 (Levenberg-Marquardt,LM) +通用全局优化算法 (Universal Global Optimization, UGO)” 对观测数据相似性关 系进行拟合. 该算法是解非线性方程的首选算法,求解能力总体上较其他算法强. 


\section{2 结果分析}

\section{1 观测点主风向及大气稳定度}

图 2a 给出了 2016 和 2017 年 4-10 月来自羊卓雍错湖面方向的风玫瑰图. 主风向为 WSW(占 20.3\%), 其次为 $\mathrm{SW}(15.9 \%)$. 湖泊大气平均压强为 $601.9 \mathrm{hPa}$ 、温度为 $8.8^{\circ} \mathrm{C}$ 、相对湿度为 $52.0 \%$. 大气静力稳定度小 于 0 的时刻高达 $81.8 \%$, 其概率密度分布呈准正态、向负 $x$ 轴偏移 (图 2b), 峰值在-0.05 0 区间 (占 $37.1 \%$ ). 稳定度的正负值频次存在日变化, 上午大气不稳定的频次明显偏多 (图 2c).
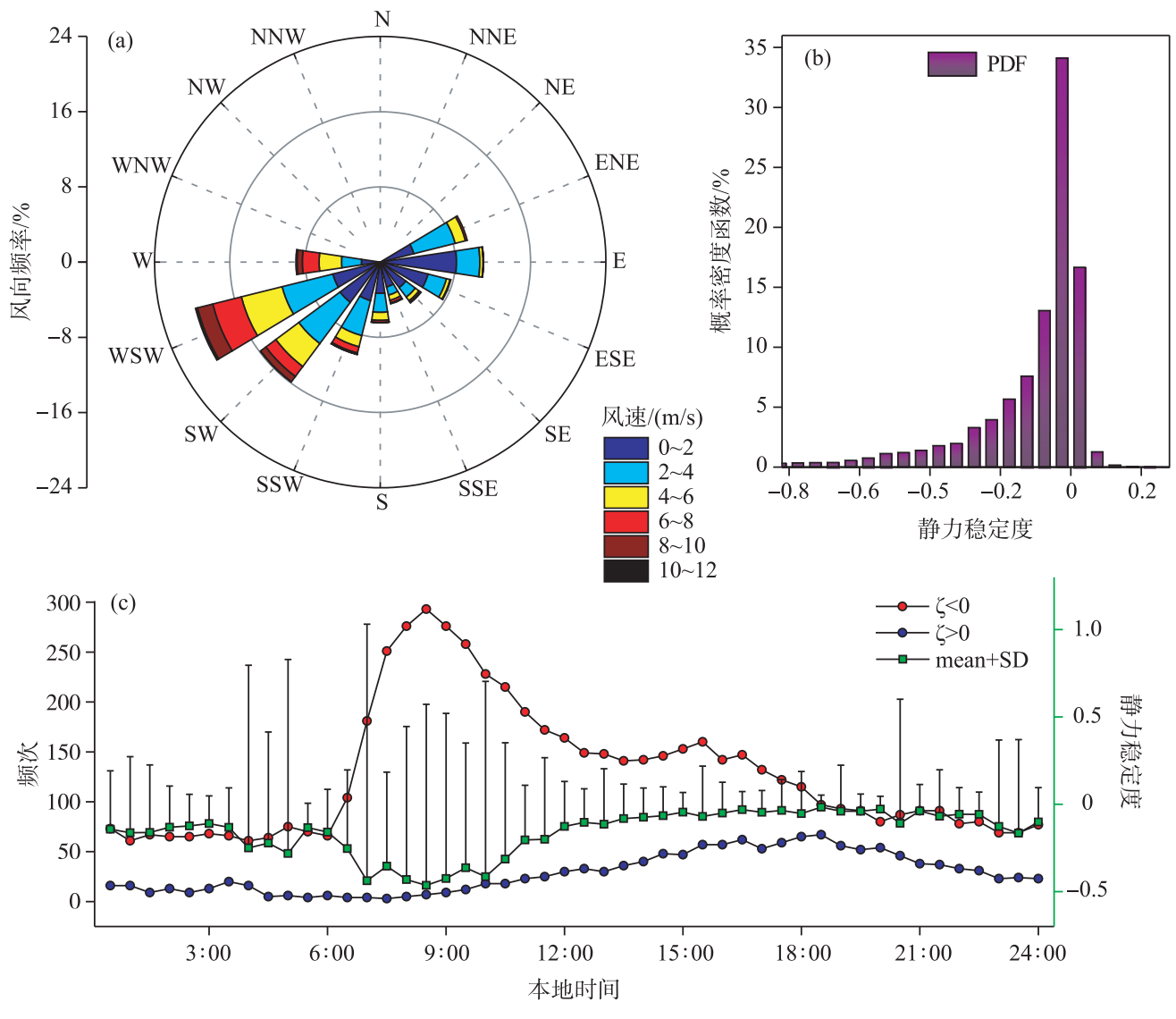

图 22016 年和 2017 年 4-10月羊卓雍错湖面风速风向玫瑰图 (a)、 静力稳定度的概率密度函数 (b) 及日变化 (c)

Fig.2 The distribution of wind speed and direction (a), the probability density function (b) , and the diurnal variations (c) of stability in Yamzhog Yumco during April-October 2016 and 2017

\section{2 湍流方差统计特征}

2.2.1 不稳定层结 不稳定层结下, 风速分量 $(u 、 v 、 w)$ 和标量 $\left(T_{\mathrm{s}} 、 d_{\mathrm{H}_{0} 0} 、 d_{\mathrm{CO}_{2}}\right)$ 无量纲标准差 $\left(\sigma_{u} /\left|u_{*}\right|\right.$ 、 $\left.\sigma_{v} /\left|u_{*}\right| 、 \sigma_{w} /\left|u_{*}\right| 、 \sigma_{t} /\left|t_{*}\right| 、 \sigma_{\mathrm{h}} /\left|h_{*}\right| 、 \sigma_{\mathrm{c}} /\left|c_{*}\right|\right)$ 随稳定度 $(\zeta)$ 的变化均符合“1/3”或“-1/3”次幂律(公 式 4) (图 3). 其中 $w$ 的拟合效果最好, 拟合曲线与原标准差数据的相关系数达 $0.89 ; d_{\mathrm{CO}_{2}}$ 拟合效果相比较差 (相关系数为 0.23 ). 不稳定层结下各变量的最优拟合表达式为: 


$$
(\zeta<0)\left\{\begin{array}{l}
\sigma_{\mathrm{u}} /\left|u_{*}\right|=3.54(1-3.28 \zeta)^{\frac{1}{3}} \\
\sigma_{\mathrm{v}} /\left|u_{*}\right|=3.91(1-2.16 \zeta)^{\frac{1}{3}} \\
\sigma_{\mathrm{w}} /\left|u_{*}\right|=0.76(1-13.52 \zeta)^{\frac{1}{3}} \\
\sigma_{\mathrm{t}} /\left|t_{*}\right|=1.32(0.0005-\zeta)^{-\frac{1}{3}} \\
\sigma_{\mathrm{h}} /\left|h_{*}\right|=1.23(0.0058-\zeta)^{-\frac{1}{3}} \\
\sigma_{\mathrm{c}} /\left|c_{*}\right|=2.15(0.0130-\zeta)^{-\frac{1}{3}}
\end{array}\right.
$$

垂直风速标准差的相似性优于水平风速已被多数观测证实 ${ }^{[22,25]}$, 说明地形起伏及下垫面物理特性差异 对垂直湍流的影响较小. 地形的作用主要影响水平方向的湍流运动, 因为水平方向的脉动主要由 “大” 的准 水平湍流产生, 往往“记忆” 着上风方向的地形特征 ${ }^{[42]}$. 此外, 在不稳定层结下,标量无量纲标准差会随着静 力不稳定度的增大而明显减小 (图 $3 \mathrm{~d} 、 \mathrm{e} 、 \mathrm{f}$ ). 原因是, 随不稳定度增加, 热力湍流运动加强, 空气间热交换加 快, 导致 $T_{\mathrm{s}} 、 d_{\mathrm{H}_{2} \mathrm{O}}$ 和 $d_{\mathrm{CO}_{2}}$ 脉动量变小; 而近地层近似为常通量层, 物理要素的特征量 $\left(t_{*} 、 h_{*} 、 c_{*}\right)$ 随高度几乎 不变,其结果是这些标量的无量纲标准差变小 ${ }^{[25]}$.

2.2.2 稳定层结 稳定层结下, $d_{\mathrm{CO}_{2}}$ 的无量纲标准差与 $\zeta$ 关系不明显, 其余变量 $\left(u 、 v 、 w 、 T_{\mathrm{s}}\right.$ 和 $\left.d_{\mathrm{H}_{2} \mathrm{O}}\right)$ 无量纲标 准差随稳定度变化基本符合“ $1 / 3$ ”或“- $1 / 3$ ”次幂律 ( 公式 4 ) (图 4). 各变量最优拟合表达式为:

$$
(\zeta>0)\left\{\begin{array}{l}
\sigma_{\mathrm{u}} /\left|u_{*}\right|=3.53(1+11.97 \zeta)^{\frac{1}{3}} \\
\sigma_{\mathrm{v}} /\left|u_{*}\right|=3.86(1+9.38 \zeta)^{\frac{1}{3}} \\
\sigma_{\mathrm{w}} /\left|u_{*}\right|=0.79(1+18.33 \zeta)^{\frac{1}{3}} \\
\sigma_{\mathrm{t}} /\left|t_{*}\right|=3.05(0.0021+\zeta)^{-\frac{1}{3}} \\
\sigma_{\mathrm{h}} /\left|h_{*}\right|=1.52(0.0201+\zeta)^{-\frac{1}{3}} \\
\sigma_{\mathrm{c}} /\left|c_{*}\right|=5.43(0.0542+\zeta)^{-\frac{1}{3}}
\end{array}\right.
$$

对比图 3 和图 4 能发现, 各变量一一尤其标量 $T_{\mathrm{s}} 、 d_{\mathrm{H}_{2} \mathrm{O}} 、 d_{\mathrm{CO}_{2}}$, 在稳定层结下的标准差数值分布比较离 散, 且拟合效果也不如不稳定层结的结果. $\mathrm{Li}$ 等 ${ }^{[26]}$ 和刘辉志等 ${ }^{[27]}$ 的研究也揭示不稳定层结下相似性关系较 好, 而稳定层结下关系消失. 其原因可能是稳定度增加到某种程度, 大气波动、间歇湍流和蜿蜒运动等导致 其偏离相似规律.

2.2.3 近中性层结 大气层结接近中性时 $(|\zeta| \rightarrow 0)$, 近地层湍流主要由机械湍流生成, 热力作用几乎没有, 此时风速分量的无量纲标准差近似等于常数 ${ }^{[43]}$. 本文将 $\zeta<0$ 的数据取绝对值, 合并 $\zeta>0$ 的数据后重新拟 合, 取 $|\zeta|=0$ 得到逼近常数: $\sigma_{\mathrm{u}} /\left|u_{*}\right|=3.57 、 \sigma_{\mathrm{v}} /\left|u_{*}\right|=3.93 、 \sigma_{\mathrm{w}} /\left|u_{*}\right|=0.77 、 \sigma_{\mathrm{t}} /\left|t_{*}\right|=20.91$ 、 $\sigma_{\mathrm{h}} /\left|h_{*}\right|=6.35 、 \sigma_{\mathrm{c}} /\left|c_{*}\right|=11.96$. 本文所得大气中性时风速分量无量纲标准差的逼近常数与青藏高原线 布河谷、理塘、玛曲等多地研究结果相近 ${ }^{[23,27,29]}$. 高原地区近中性层结下水平风速标准差普遍大于、垂直风 速标准差普遍小于戈壁、平原等地区的拟合值 ${ }^{[44-45]}$, 可能是由于高原地区常年风速较大所致 ${ }^{[23]}$. 而拟合系 数小于沙漠湖泊和海洋结果 ${ }^{[15,46]}$, 可能是受局地环境 ${ }^{[15]}$ 或观测高度 ${ }^{[7]}$ 影响, 尚待进一步深人研究.

\section{3 湍流特征量的统计和变化特征}

2.3.1 湍流强度 2016 和 2017 年 4-10月 $u 、 v$ 和 $w 3$ 个方向的湍流强度 $\left(I_{\mathrm{u}} 、 I_{\mathrm{v}}\right.$ 和 $\left.I_{\mathrm{w}}\right)$ 多数时刻在 0.5 以 内, 分别占 $61.8 \% 、 64.3 \%$ 和 $98.4 \%$, 满足泰勒假说的前提条件, 即认为湍涡发展的时间尺度大于其平移过传 感器的时间 ${ }^{[47]}$. 其概率密度分布显示, $I_{\mathrm{u}} 、 I_{\mathrm{v}}$ 和 $I_{\mathrm{w}}$ 最集中的区间分别是 $0.2 \sim 0.3(20.5 \%) 、 0.2 \sim 0.3(22.2 \%)$ 和 $0 \sim 0.1(60.3 \%)$; 水平方向的湍流强度 $\left(I_{\mathrm{u}}\right.$ 和 $I_{\mathrm{v}}$ 的平均值分别为 0.60 和 0.58$)$ 大于垂直方向 $\left(I_{\mathrm{w}}\right.$ 的平均值为 0.13 ), 数值的离散性也更大 ( 图 $5 \mathrm{a})$.

$I_{\mathrm{u}} 、 I_{\mathrm{v}}$ 和 $I_{\mathrm{w}}$ 均在 $U<1 \mathrm{~m} / \mathrm{s}$ 的微风环境中最强(平均值分别为 $1.41 、 1.40$ 和 0.31 ) (图 $5 \mathrm{~b}$ ), 这是因为在微 风环境中大气运动受温度差主导, 即浮力作用导致湍流, 产生对流运动, 边界层大气处于通常所说的自由对 流状态, 此时湍流发展最为旺盛. 随着 $U$ 增大, $I_{\mathrm{u}} 、 I_{\mathrm{v}}$ 和 $I_{\mathrm{w}}$ 均明显减弱, 但减弱速度逐渐放缓; $U>5 \mathrm{~m} / \mathrm{s}$ 后基 

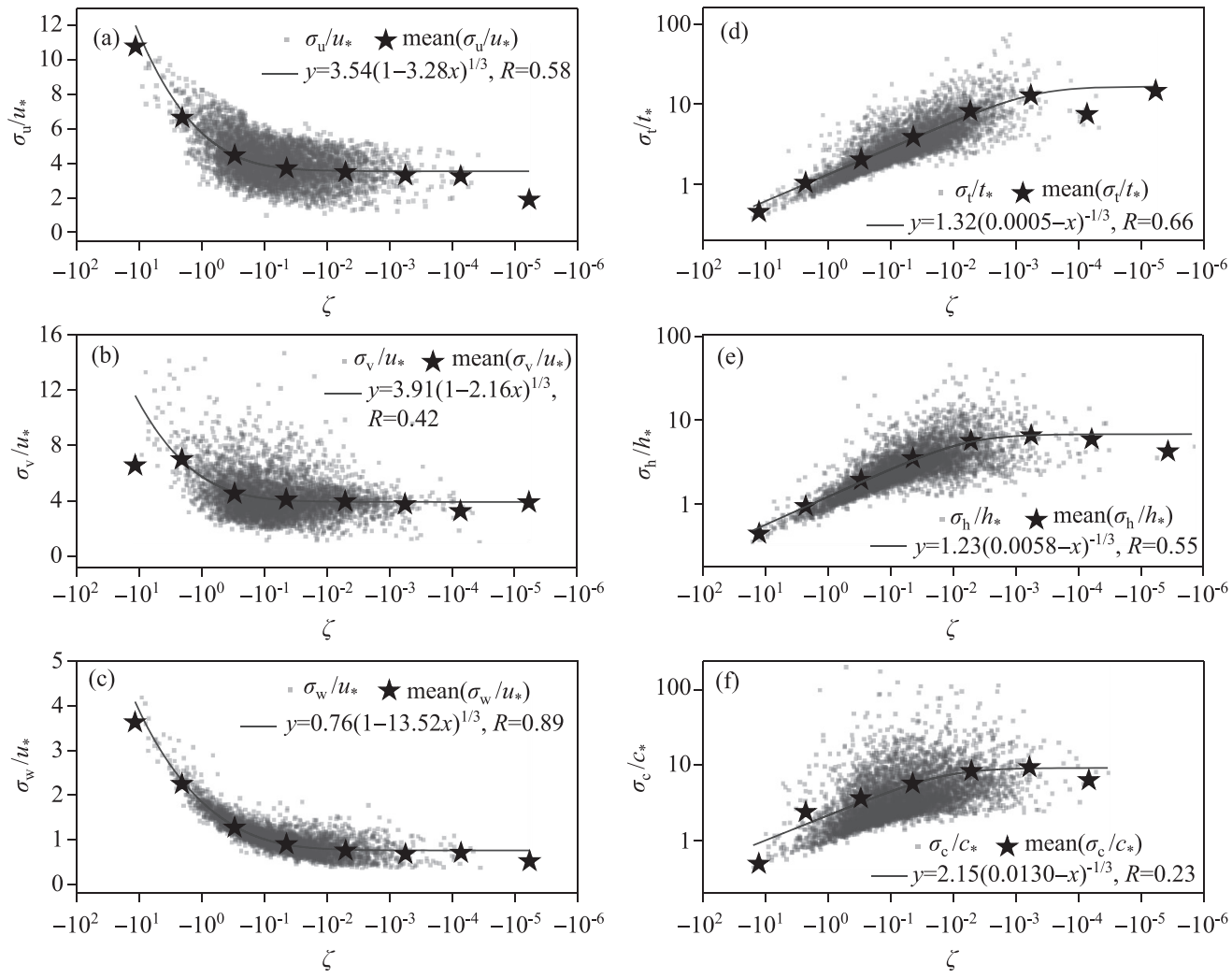

图 32016 年和 2017 年 4-10 月羊卓雍错湖面不稳定大气层结时 $u(\mathrm{a}) 、 v(\mathrm{~b}) 、 w(\mathrm{c}) 、$

$T_{\mathrm{s}}(\mathrm{d}) 、 d_{\mathrm{H}_{2} \mathrm{O}}(\mathrm{e}) 、 d_{\mathrm{CO}_{2}}(\mathrm{f})$ 的无量纲标准差随稳定度的变化(五角星代表每个区段的平均值,

区段分别取 $-100 \sim-10 \sim-1 \sim-0.1 \sim-0.01 \sim-0.001 \sim-0.0001 \sim-0.00001)$

Fig.3 Variations of the dimensionless standard deviations of $u(\mathrm{a}), v(\mathrm{~b}), w(\mathrm{c}), T_{\mathrm{s}}(\mathrm{d}), d_{\mathrm{H}, 0}(\mathrm{e})$ and $d_{\mathrm{CO}_{2}}(\mathrm{f})$ with $\zeta$ in unstable stratification in Yamzhog Yumco during April-October 2016 and 2017

(The five-pointed stars represent the mean values of dimensionless quantities and $\zeta$ in each section of $-100--10--1--0.1--0.01--0.001--0.0001--0.00001$ )

本不再随风速变化. 根据计算公式 (1), $I$ 的大小取决于风速分量标准差 $\left(\sigma_{\mathrm{u}} 、 \sigma_{\mathrm{v}}\right.$ 和 $\left.\sigma_{\mathrm{w}}\right)$ 和平均风速 $(U)$; $I_{\mathrm{u}} 、 I_{\mathrm{v}}$ 和 $I_{\mathrm{w}}$ 均随 $U$ 增大而减小 (图 $5 \mathrm{~b}$ ), 并呈显著负相关性 $(R=-0.39 、-0.42 、-0.34$ ), 充分说明平均风速的变 化幅度大于风速方差变化幅度.

2.3.2 湍流动能 大气湍流运动的发生、发展、维持、衰减或消逝, 归根到底是取决于湍流动能( TKE ) 增加、 不变或减少的结果 ${ }^{[48]}$. 根据 $T K E$ 的公式(2), TKE 的大小由 3 个风速分量的脉动方差决定. $U$ 越大, 风速分 量波动幅度也大, 导致 $T K E$ 越大. 张宏升等 ${ }^{[49]}$ 与李锁锁等 ${ }^{[30]}$ 的观测均表明 $T K E$ 随 $U$ 增大而增大. 本文结果 显示, 湖面大气 $T K E$ 不仅与 $U$ 呈正相关, 而且二者存在较好的线性关系 (图 6a) : $T K E=0.45 U+0.40, R=0.50$.

$T K E$ 同时受大气层结的影响, 大气接近中性时 $T K E$ 越强, 其中 $T K E>10 \mathrm{~m}^{2} / \mathrm{s}^{2}$ 的时刻 $\zeta$ 全部位于 $-0.1 \sim$ 0.1 范围内. 随大气越稳定或者越不稳定, $T K E$ 皆减弱 (图 6b), 是因为不同层结下为湍流补充动能的驱动力 不同. 从 TKE 方程可知, 维持浴旋发展的能量主要来自风切变项和浮力项, 前者基本比后者大一个数量级, 是产生 TKE 的主要来源 ${ }^{[50]}$. 大气层结近中性时, 唯一或主要的湍流能量产生机制是剪切作用, 与之有关的 是风剪切和表面应力, 浮力作用极小; 层结不稳定时, 浮力成为驱动湍流的主要机制, 即热力驱动是能量的 主要来源, 而非动力驱动; 层结稳定时, 因有逆温层, 浮力作用不但无法为湍流补给动能, 反而起到抑制或削 弱作用 ${ }^{[20]}$. 

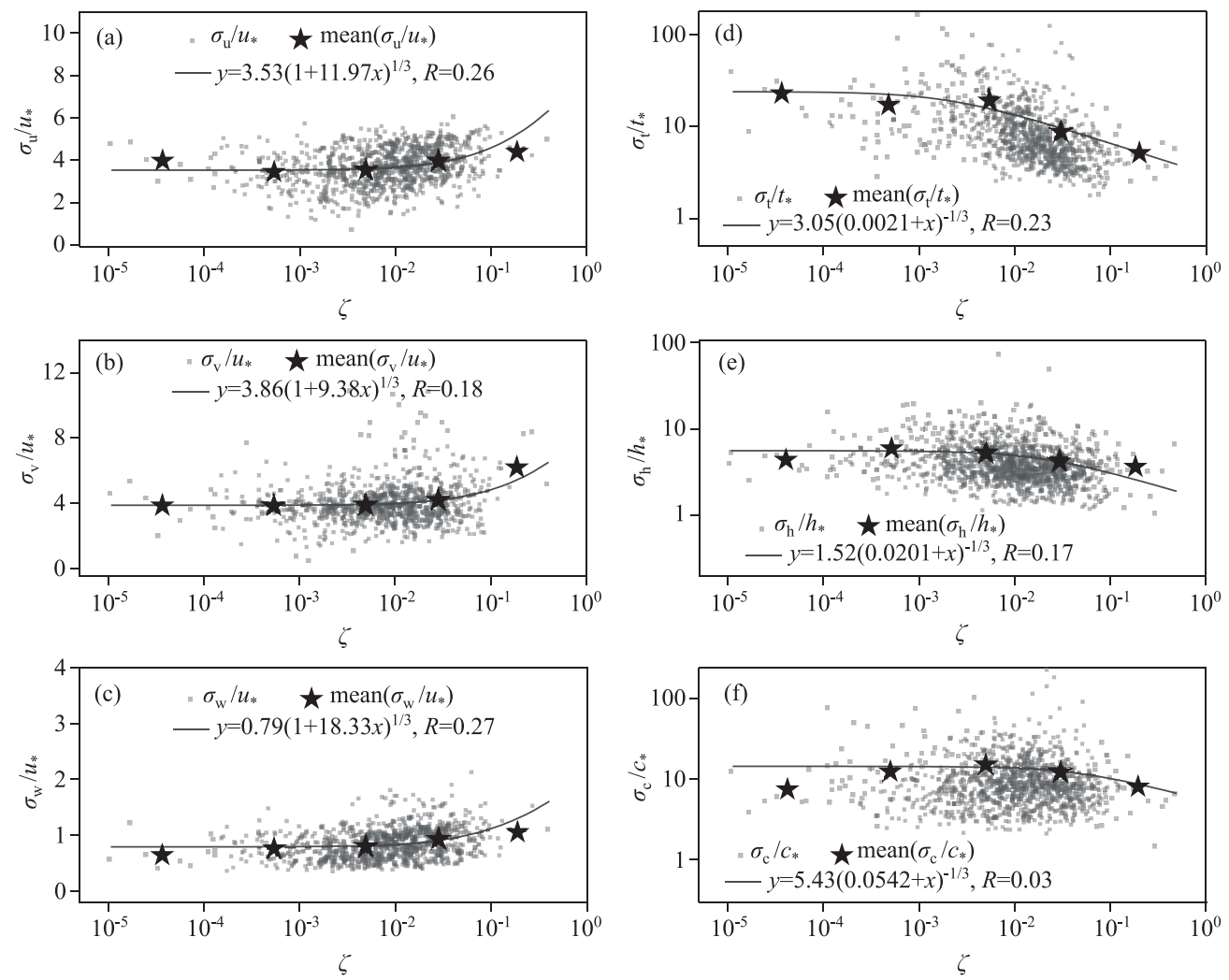

图 42016 年和 2017 年 4-10 月羊卓雍错湖面稳定大气层结时 $u(\mathrm{a}) 、 v(\mathrm{~b}) 、 w(\mathrm{c}) 、 T_{\mathrm{s}}(\mathrm{d}) 、$ $d_{\mathrm{H}_{2} \mathrm{O}}(\mathrm{e}) 、 d_{\mathrm{CO}_{2}}$ (f) 的无量纲标准差随稳定度的变化(五角星代表每个区段的平均值, 区段分别取 $0.00001 \sim 0.0001 \sim 0.001 \sim 0.01 \sim 0.1 \sim 1 \sim 10)$

Fig.4 Variations of the dimensionless standard deviations of $u(\mathrm{a}), v(\mathrm{~b}), w(\mathrm{c}), T_{\mathrm{s}}$ (d), $d_{\mathrm{H}_{2} \mathrm{O}}(\mathrm{e})$ and $d_{\mathrm{CO}_{2}}(\mathrm{f})$ with $\zeta$ in stable stratification in Yamzhog Yumco during April-October 2016 and 2017 ( The five-pointed stars represent the mean values of dimensionless quantities and $\zeta$ in each section of $0.00001-0.0001-0.001-0.01-0.1-1-10$ )

随着层结稳定或不稳定程度加剧, 无量纲湍流动能 $\left(T K E / u_{*}{ }^{2}\right)$ 的数值及离散性不断增大 (图 $\left.6 \mathrm{c} 、 \mathrm{~d}\right)$, 且 变化符合“ $1 / 3$ ” 次幂律 (公式(4)), 最优拟合式为:

$$
\begin{cases}T K E / u_{*}^{2}=13.81(1-17.14 \zeta)^{\frac{1}{3}} & (\zeta<0) \\ T K E / u_{*}^{2}=14.73(1+32.55 \zeta)^{\frac{1}{3}} & (\zeta>0)\end{cases}
$$

2.3.3 湍流通量 羊卓雍错湖面的动量通量、感热通量和潜热通量均存在明显日变化. 动量通量夜间逐渐减 弱, 8:30 达到最低值 $0.022 \mathrm{~kg} /\left(\mathrm{m} \cdot \mathrm{s}^{2}\right)$, 然后迅速增强, 15:00 时达 $0.109 \mathrm{~kg} /\left(\mathrm{m} \cdot \mathrm{s}^{2}\right)$, 下午到傍晚保持高值 (图 7). 动量通量对风速有强烈依赖性 ${ }^{[51]}$. 观测点下午到傍晚多处于大风环境, 使得湖面粗鋉度偏大, 动力 湍流增加, 大气向湖面输送动量增强. 4-10 月 30 min 动量通量与风速和风速平方均有较好的相关性, 相关 系数分别达 0.32 和 0.33 , 动量通量与风速平方的拟合回归方程式为: $y=0.002 x+0.039$ (图 $8 \mathrm{a}$ ).

羊卓雍错湖面热量输送以潜热为主, $4-10$ 月潜热通量日平均值 $\left(77.3 \mathrm{~W} / \mathrm{m}^{2}\right)$ 是感热通量 $\left(14.6 \mathrm{~W} / \mathrm{m}^{2}\right)$ 的 5.3 倍. 感热和潜热通量日变化均表现为单波峰日变化规律, 感热通量夜间逐渐增强, $5: 30$ 达到峰值 22.4 $\mathrm{W} / \mathrm{m}^{2}, 18: 00$ 降到谷值 $6.1 \mathrm{~W} / \mathrm{m}^{2}$; 潜热通量在 7:00 时最低 $\left(51.4 \mathrm{~W} / \mathrm{m}^{2}\right), 16: 00$ 时最高 $\left(106.6 \mathrm{~W} / \mathrm{m}^{2}\right)$ (图 7). 

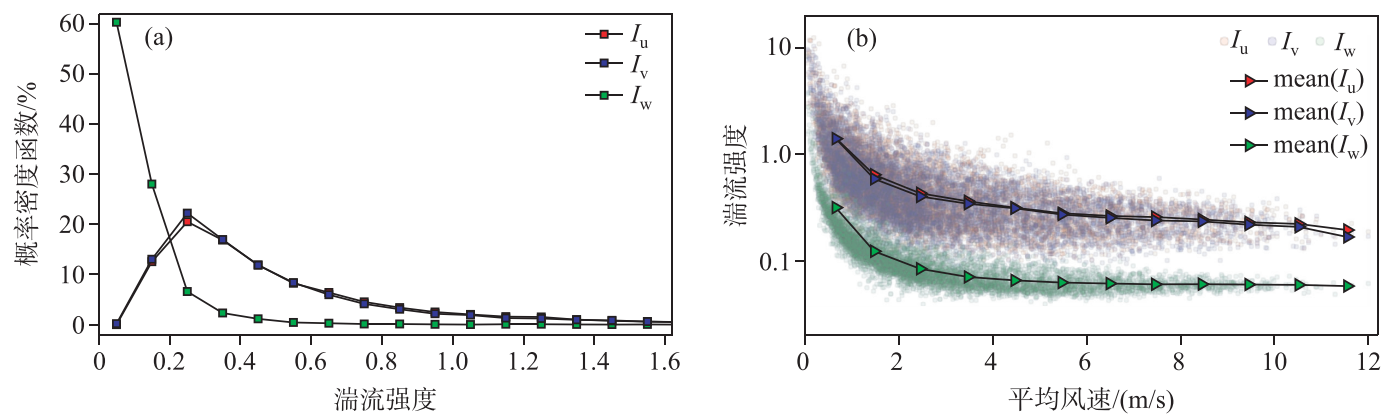

图 52016 年和 2017 年 4-10 月羊卓雍错湖面湍流强度的概率密度函数 (a) 和随平均风速的变化(b) (b 中的点线代表每个区段内湍流强度和风速的平均值, 区段长度取风速 $1 \mathrm{~m} / \mathrm{s}$ )

Fig.5 Probability density functions of three-dimensional turbulent intensities (a) and variations of three-dimensional turbulent intensities with average wind speed (b) in Yamzhog Yumco during April-October 2016 and 2017 (The dot lines in (b) represent the mean values of turbulent intensities and wind speed in each wind speed section with length $1 \mathrm{~m} / \mathrm{s}$ )
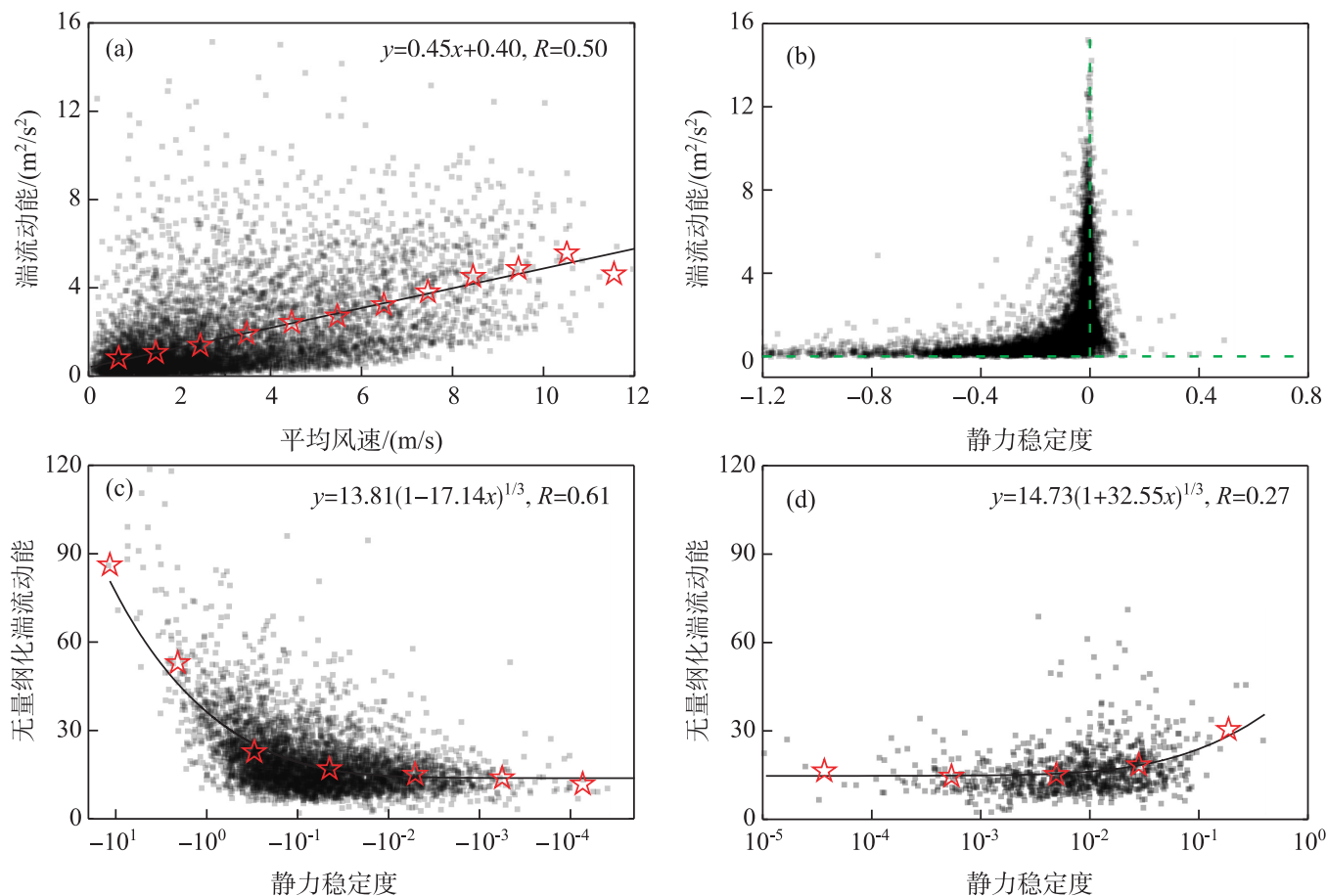

图 62016 年和 2017 年 4-10 月羊卓雍错湖面湍流动能随平均风速 (a)、

稳定度 $(b)$ 的变化, 以及无量纲化湍流动能随稳定度的变化 $(\mathrm{c} 、 \mathrm{~d})$ （ a 中五角星代表 每个区段平均值, 区段长度取风速 $1 \mathrm{~m} / \mathrm{s}, \mathrm{c} 、 \mathrm{~d}$ 中的五角星代表每个区段的平均值, 区段

分别取 $-10 \sim-1 \sim-0.1 \sim-0.01 \sim-0.001 \sim-0.0001 \sim-0.00001$ 和 $0.00001 \sim 0.0001 \sim 0.001 \sim 0.01 \sim 0.1 \sim 1 \sim 10$ )

Fig. 6 Variations of turbulent kinetic energy with average wind speed (a), stability (b), and the variations of dimensionless turbulent kinetic energy with stability in unstable stratification (c)

and stable stratification (d) in Yamzhog Yumco during April-October 2016 and 2017

( The five-pointed stars in (a) represent the mean values of turbulent kinetic energy and wind speed in each wind speed section with length $1 \mathrm{~m} / \mathrm{s}$; The five-pointed stars in (c) and

(d) represent the mean values of dimensionless turbulent kinetic energy and stability in the each section of $-10--1--0.1--0.01--0.001--0.0001--0.00001$ and $0.00001-0.0001-0.001-0.01-0.1-1-10$ ) 
感热和潜热通量的峰值分别出现在清晨和下午, 与 Guo 等 ${ }^{[31]}$ 、 Liu 等 ${ }^{[14]}$ 研究色林错和洱海所得结果一致. 湖 面感热通量与湖一气温差、风速与湖一气温差的乘积存在显著正相关 $(R=0.63 、 0.64)$, 但与风速的相关性不 明显 $(R=-0.08)$, 这与青海湖 ${ }^{[31]}$ 和太湖 ${ }^{[52]}$ 的结论 $(R$ 分别为 0.27 和 0.36$)$ 有所不同. 湖面潜热通量与风速、 饱和水汽压差以及风速和饱和水汽压差的乘积均存在较好的相关性 $(R=0.63 、 0.34 、 0.62)$. 拟合结果显示, 感热通量与风速和湖一气温差乘积的线性方程为 $y=0.8 x+15.1$, 潜热通量与风速和饱和水汽压差乘积的线 性方程为 $y=1.6 x+50.4$ (图 8b、c).

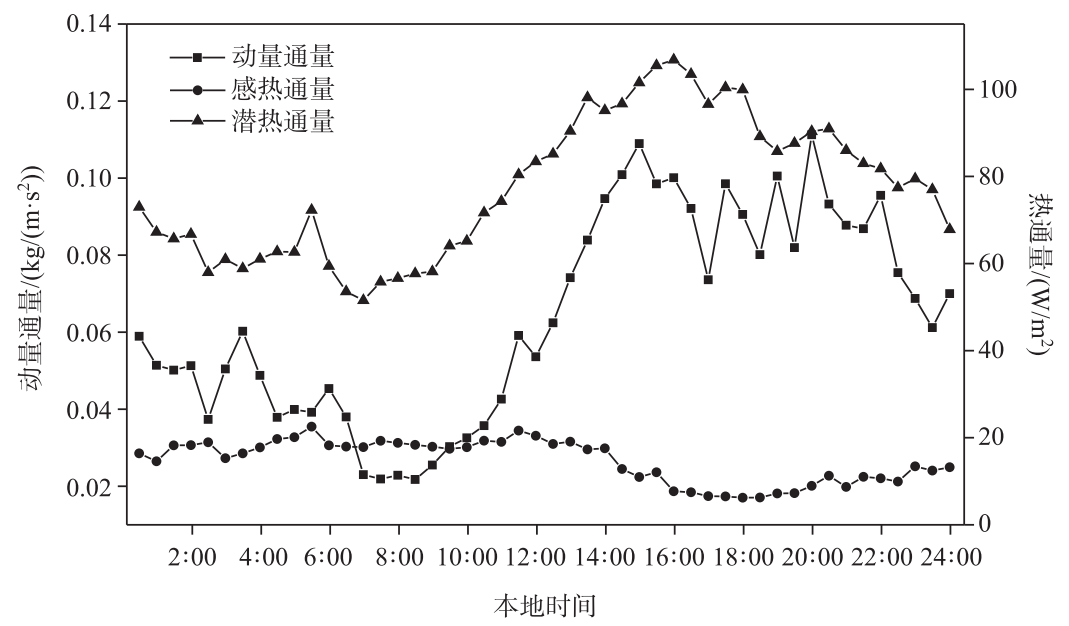

图 72016 年和 2017 年 4-10 月羊卓雍错湖面动量通量、感热通量和潜热通量的日变化

Fig.7 Diurnal variations of momentum flux, sensible heat flux and latent heat flux in Yamzhog Yumco during April-October 2016 and 2017
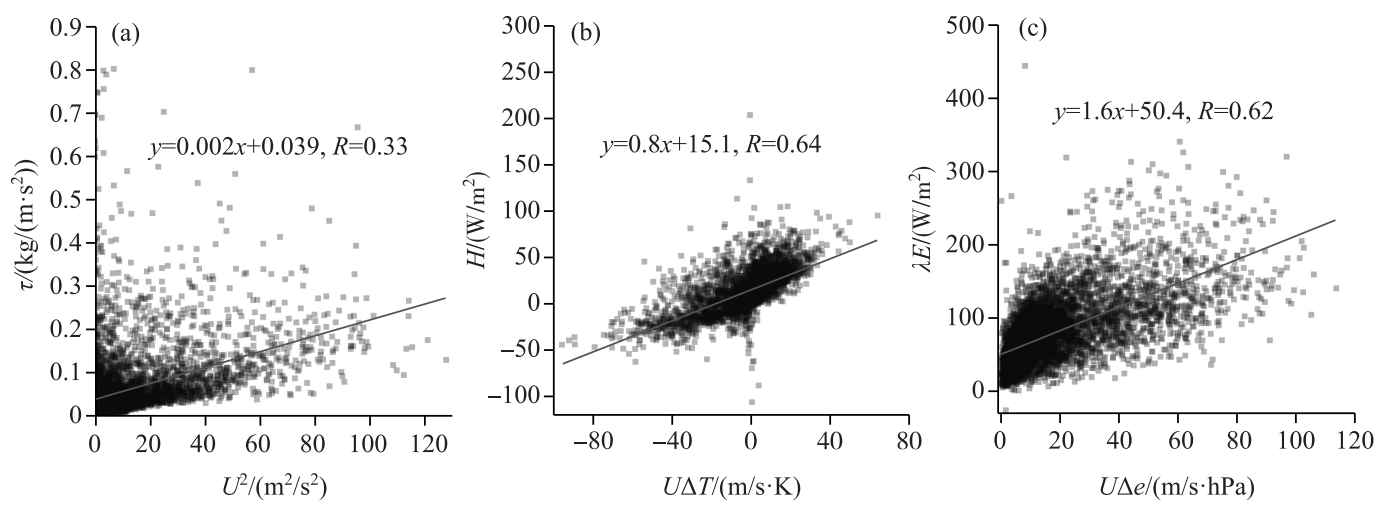

图 82016 年和 2017 年 4-10月羊卓雍错湖面动量通量与风速平方 ( a)、感热通量与风速和 湖一气温差 $(\Delta T)$ 的乘积 $(\mathrm{b})$ 以及潜热通量与风速和饱和水汽压差 $(\Delta e)$ 的乘积 $(\mathrm{c})$ 的关系

Fig. 8 Relationships between momentum flux and the square value of wind speed (a), sensible heat flux and the product of wind speed and difference in temperature $(\Delta T)$ between the water surface and overlying air $(\mathrm{b})$, and latent heat flux and the product of wind speed and difference in vapor pressure $(\Delta e)$ between water-air interface and overlying air (c) in Yamzhog Yumco during April-October 2016 and 2017

\section{3 结论}

通过分析 2016 和 2017 年 4-10 月表征羊卓雍错湖泊的浴动观测数据, 揭示该湖泊湖面风速分量无量 
纲标准差随稳定度的变化满足 Monin-Obukhov 相似理论的“ $1 / 3$ ”次幂律, 拟合效果为 $w>u>v$ 方向, 当大气 层结近中性时 $\sigma_{\mathrm{u}} /\left|u_{*}\right|=3.57 、 \sigma_{\mathrm{v}} /\left|u_{*}\right|=3.93 、 \sigma_{\mathrm{w}} /\left|u_{*}\right|=0.77$. 超声虚温、水汽和 $\mathrm{CO}_{2}$ 密度等标量仅在 不稳定层结下服从 “ $-1 / 3$ ” 次幂律, 稳定条件下拟合效果较差, 尤其 $\mathrm{CO}_{2}$ 密度的无量纲标准差与稳定度无明 显关系. 羊卓雍错湖面大气的水平湍流强度较垂直方向强 $\left(I_{\mathrm{u}} \approx I_{\mathrm{v}}>I_{\mathrm{w}}\right), I_{\mathrm{u}} 、 I_{\mathrm{v}}$ 和 $I_{\mathrm{w}}$ 均随平均风速增大而减 弱, 减弱速度逐渐放缓. 湖面湍流动能随风速增大而增大, 而且呈显著线性变化关系 (增长率达 $0.45 \mathrm{~m} / \mathrm{s}$ ). 湖面动量通量在下午和傍晚输送较强, 热通量输送以潜热为主、向上输送, 感热和潜热通量的峰值分别出现 在清晨和下午. 动量通量与风速的平方、感热通量与风速和湖一气温差的乘积以及潜热通量与风速和饱和水 汽压差的乘积均存在显著正相关关系.

致谢: 感谢西藏白地水文站米玛次仁、天津师范大学者萌及北京天诺基业科技有限公司杨超、孔维维等先生 在野外仪器安装和维护等诸多方面给予的悉心指导与鼎力相助.

\section{4 参考文献}

[ 1 ] Song CQ, Huang B, Ke LH et al. Seasonal and abrupt changes in the water level of closed lakes on the Tibetan Plateau and implications for climate impacts. Journal of Hydrology, 2014, 514: 131-144.

[ 2 ] Xiao W, Liu SD, Li XH et al. Transfer coefficients of momentum, heat and water vapour in the atmospheric surface layer of a large shallow freshwater lake: A case study of Lake Taihu. J Lake Sci, 2012, 24(6) : 932-942. DOI: 10.18307/ 2012.0617. [肖薇, 刘寿东, 李旭辉等. 大型浅水湖泊与大气之间的动量和水热交换系数——以太湖为例. 湖泊科 学, 2012, 24(6): 932-942.]

[ 3 ] Long Z, Perrie W, Gyakum J et al. Northern lake impacts on local seasonal climate. Journal of Hydrometeorology, 2007, 8 (4) : 881-896.

[ 4 ] Flagg D, Brook J, Sills D et al. Lake Breezes in Southern Ontario: Observations, models and impacts on air quality. In: Borrego C, Miranda AI eds. Air pollution modeling and its application XIX. NATO Science for Peace and Security Series Series C: Environmental Security. Dordrecht: Springer, 2008: 679-680.

[ 5 ] Samuelsson P, Kourzeneva E, Mironov D. The impact of lakes on the European climate as simulated by a regional climate model. Boreal Environment Research, 2010, 15(2) : 113-129.

[ 6 ] Wang W. Energy budget at Lake Taihu and its response to climate change [Dissertation]. Nanjing: Nanjing University of Information Science and Technology, 2014: 1-3. [王伟. 太湖能量收支及其对气候变化的响应 [学位论文]. 南京: 南 京信息工程大学, 2014: 1-3.]

[ 7 ] Xiao W, Liu SD, Wang W et al. Transfer coefficients of momentum, heat and water vapour in the atmospheric surface layer of a large freshwater lake. Boundary-Layer Meteorology, 2013, 148(3) : 479-494.

[ 8 ] Wang W, Xiao W, Cao C et al. Temporal and spatial variations in radiation and energy balance across a large freshwater lake in China. Journal of Hydrology, 2014, 511: 811-824.

[ 9 ] Lee XH, Liu SD, Xiao W et al. The Taihu Eddy Flux Network: An observational program on energy, water, and greenhouse gas fluxes of a large freshwater lake. Bulletin of the American Meteorological Society, 2014, 95. DOI : 10.1175/ BAMS-D-13-00136.1.

[10] Zhao XS, Wang SG, Li M et al. Energy flux measurements and environmental controls in summer over the Poyang Lake, China. J Lake Sci, 2014, 26(6) : 955-962. DOI: 10.18307/2014.0619. [赵晓松, 王仕刚, 李梅等. 鄱阳湖夏季水热 通量特征及环境要素影响分析. 湖泊科学, 2014, 26(6) : 955-962.]

[11] Zhao XS, Liu YB. Phase transition of surface energy exchange in China's largest freshwater lake. Agricultural and Forest Meteorology, 2017, 244/245: 98-110.

[12] Wang BB, Ma YM, Chen XL et al. Observation and simulation of lake-air heat and water transfer processes in a high-altitude shallow lake on the Tibetan Plateau. Journal of Geophysical Research: Atmospheres, 2015, 120(24) : 12327-12344.

[13] Wang BB, Ma YM, Ma WQ et al. Physical controls on half-hourly, daily, and monthly turbulent flux and energy budget over a high-altitude small lake on the Tibetan Plateau. Journal of Geophysical Research: Atmospheres, 2017, 122(4): 2289-2303.

[14] Liu HZ, Feng JW, Sun JH et al. Eddy covariance measurements of water vapor and $\mathrm{CO}_{2}$, fluxes above the Erhai Lake. Science China: Earth Sciences, 2015, 58(3): 317-328. 
[15] Hu WF, Wang NA, Zhao LQ et al. Water-heat exchange over a typical lake in Badain Jaran Desert, China. Progress in Geography, 2015, 34(8): 1061-1071. [胡文峰, 王乃昂, 赵力强等. 巴丹吉林沙漠典型湖泊湖气界面水一热交换特 征. 地理科学进展, 2015, 34(8): 1061-1071.]

[16] Nordbo A, Launiainen S, Mammarella I et al. Long-term energy flux measurements and energy balance over a small boreal lake using eddy covariance technique. Journal of Geophysical Research: Atmospheres, 2011, 116 ( D2 ). DOI: 10. 1029/2010JD014542.

[17] Wang DD, Wang W, Liu SD et al. Characteristics of modeling hourly water surface evaporation in Lake Taihu and comparison of simulation results by three models. J Lake Sci, 2017, 29(6) : 1538-1550. DOI: 10.18307/2017.0626. [王丹丹, 王伟, 刘寿东等. 太湖小时尺度水面蒸发特征及 3 种模型模拟效果对比. 湖泊科学, 2017, 29(6): 1538-1550.]

[18] Assouline S, Tyler SW, Tanny J et al. Evaporation from three water bodies of different sizes and climates: Measurements and scaling analysis. Advances in Water Resources, 2008, 31(1) : 160-172.

[19] Hu YQ. Boundary layer meteorology. Advance in Earth Sciences, 1991, 6(6): 57-59. [胡隐樵. 边界层气象学. 地球科 学进展, 1991, 6(6) : 57-59.]

[20] Sheng PX, Mao JT, Li JG et al eds. Atmospheric Physics. Beijing: Peking University Press, 2003: 239-272. [盛裴轩, 毛节泰, 李建国等. 大气物理学. 北京: 北京大学出版社, 2003: 239-272.]

[21] Monin AS, Obukhov AM. Dimensionless characteristics of turbulence in the atmospheric surface layer. Doklady Akademii Nauk SSSR, 1953, 93(2) : 223-226.

[22] Liu HZ, Hong ZX. Turbulent characteristics in the surface layer over Gerze Area in the Tibetan Plateau. Scientia Atmospherica Sinica, 2000, 24(3) : 289-300. [刘辉志, 洪钟祥. 青藏高原改则地区近地层湍流特征. 大气科学, 2000, 24(3) : 289-300.]

[23] Chen YG, Zhang Y, Wang SY et al. Seasonal variation of turbulence characteristics over alpine meadow ecosystem. Plateau Meteorology, 2014, 33(3) : 585-595. [ 陈云刚, 张宇, 王少影等. 高寒草甸湍流特征量的季节变化特征. 高原气 象, 2014, 33(3): 585-595.]

[24] Yang LW, Gao XQ, Hui XY et al. Study on turbulence characteristics in the atmospheric surface layer over Nyainrong grassland in central Qinghai-Tibetan Plateau. Plateau Meteorology, 2017, 36(4) : 875-885. [杨丽薇, 高晓清, 惠小英 等. 青藏高原中部聂荣亚寒带半干旱草地近地层湍流特征研究. 高原气象, 2017, 36(4) : 875-885.]

[25] Ma YM, Ma WQ, Hu ZY et al. Similarity analysis of atmospheric turbulent intensity over grassland surface of Qinghai-Xizang Plateau. Plateau Meteorology, 2002, 21(5): 514-517. [马耀明, 马伟强, 胡泽勇等. 青藏高原草甸下垫面湍流 强度相似性关系分析. 高原气象, 2002, 21(5) : 514-517.]

[26] Li MS, Ma YM, Ma WQ et al. Analysis of turbulence characteristics over the northern Tibetan Plateau area. Advances in Atmospheric Sciences, 2006, 23(4) : 579-585.

[27] Liu HZ, Feng JW, Zou H et al. Turbulent characteristics of the surface layer in Rongbuk Valley on the northern slope of Mt. Qomolangma. Plateau Meteorology, 2007, 26(6) : 1151-1161. [刘辉志, 冯健武, 邹捍等. 青藏高原珠峰线布河 谷地区近地层湍流输送特征. 高原气象, 2007, 26(6): 1151-1161.]

[28] Guo XF, Zhang HS, Cai XH et al. Flux-variance method for latent heat and carbon dioxide fluxes in unstable conditions. Boundary-Layer Meteorology, 2009, 131(3) : 363-384.

[29] Li Y, Li YQ, Zhao XB. Analysis of turbulent characteristics in the surface layer inLitang region on the east edge of Tibetan Plateau. Plateau Meteorology, 2009, 28(4) : 745-753. [李英, 李跃清, 赵兴炳. 青藏高原东坡理塘地区近地层湍流 特征研究. 高原气象, 2009, 28(4): 745-753.]

[30] Li SS, Lv SH, Gao YH et al. Analysis of the statistical characteristics of the turbulent data at Maqu area in the upper Yellow River. Advances in Earth Science, 2012, 27(8): 901-907. [李锁锁, 吕世华, 高艳红等. 黄河上游玛曲草原湍 流统计特征分析. 地球科学进展, 2012, 27(8): 901-907.]

[31] Guo YH, Zhang YS, Ma N et al. Quantifying surface energy fluxes and evaporation over a significant expanding endorheic lake in the central Tibetan Plateau. Journal of the Meteorological Society of Japan, 2016, 94(5) : 453-465.

[32] Li XY, Ma YJ, Huang YM et al. Evaporation and surface energy budget over the largest high-altitude saline lake on the Qinghai-Tibet Plateau. Journal of Geophysical Research: Atmospheres, 2016, 121(18) : 10470-10485.

[33] Li ZG, Lyu SH, Ao YH et al. Long-term energy flux and radiation balance observations over Lake Ngoring, Tibetan Plateau. Atmospheric Research, 2015, 155: 13-25. 
[34] Chu D, Pu Q, Laba ZM et al. Remote sensing analysis on lake area variations of Yamzho Yunco in Tibetan Plateau. J Lake $S c i, 2012,24(3)$ : 494-502. DOI: 10.18307/2012.0324. [ 除多, 普穷, 拉巴卓玛等. 近 40 a 西藏羊卓雍错湖泊面积 变化遥感分析. 湖泊科学, 2012, 24(3): 494-502.]

[35] LI-CORInc ed. EddyPro software instruction manual: 13th edition, 2017. https://www.licor.com/env/support/product? $\mathrm{p}=\mathrm{ec}$.

[36] Lee XH, Massman W, Law B eds. Handbook of micrometeorology: A guide for surface flux measurement and analysis. Dordrecht: Kluwer Academic Publishers, 2004: 181-208.

[37] Schmid HP, Csb G, Cropley F et al. Measurements of $\mathrm{CO}_{2}$ and energy fluxes over a mixed hardwood forest in the midwestern United States. Agricultural and Forest Meteorology, 2000, 103(4) : 357-374.

[38] Kljun N, Calanca P, Rotach MW et al. A simple parameterization for flux footprint predictions. Boundary-Layer Meteorolo$g y, 2004,112(3): 503-523$.

[39] Stull RB ed. Trans. by Yang CX. An introduction to boundary layer meteorology. Beijing: Meteorological Press, 1991: 4850, 187-191. [Stull RB 著. 杨长新译. 边界层气象学导论. 北京: 气象出版社, 1991: 48-50, 187-191.]

[40] Panofsky HA, Tennekes H, Lenschow DH et al. The characteristics of turbulent velocity components in the surface layer under convective conditions. Boundary-Layer Meteor, 1977, 11(3) : 355-361.

[41] Tillman JE. The indirect determination of stability, heat and momentum fluxes in the atmospheric boundary layer from simple scalar variables during dry unstable conditions. Journal of Applied Meteorology, 1972, 11(5) : 783-792.

[42] Zhao M, Miao MQ, Wang YC eds. Boundary meteorology. Beijing: Meteorology Press, 1991: 465. [赵鸣, 苗曼倩, 王彦 昌. 边界层气象学教程. 北京: 气象出版社, 1991: 465.]

[43] Yue P, Zhang Q, Niu SJ et al. Statistical characteristic of atmospheric turbulence in clear and dust weather conditions in Inner Mongolian Grassland during spring. Plateau Meteorology, 2011, 30(5): 1180-1188. [岳平, 张强, 牛生杰等. 春 季内蒙古草原典型晴天与沙尘条件下湍流速度统计特征对比分析. 高原气象, 2011, 30(5): 1180-1188.]

[44] Panofsky HA, Dutton JA eds. Atmospheric turbulence. Models and methods for engineering applications. New York: John Wiley \& Sons Inc., 1984.

[45] Zhang HS, Li FY, Chen JY. Statistical characteristics of atmospheric turbulence in different underlying surface conditions. Plateau Meteorology, 2004, 23(5) : 598-604. [张宏升, 李富余, 陈家宜. 不同下垫面湍流统计特征研究. 高原气 象, 2004, 23(5): 598-604.]

[46] Duan ZQ. Eddy covariance measurements of marine atmospheric boundary-layer turbulence and air- sea fluxes. Qingdao: Ocean University of China [Dissertation], 2013: 36-59. [段自强. 利用浴动相关法研究海洋大气边界层湍流特征与 海气物质交换 [学位论文]. 青岛: 中国海洋大学, 2013: 36-59.]

[47] Willis GE, Deardorff JW. On the use of Taylor's translation hypothesis for diffusion in the mixed layer. Quarterly Journal of the Royal Meteorological Society, 1976, 102(434) : 817-822.

[48] Yu ZH, Miao MQ, Jiang QR et al eds. Fluid mechanics: 3rd edition. Beijing: Meteorology Press, 2004: 212-218. [余志 豪, 苗曼倩, 蒋全荣等. 流体力学: 第三版. 北京: 气象出版社, 2004: 212-218.]

[49] Zhang HS, Liu XJ, Zhu H et al. Characteristics of turbulent transfer during the strong wind period in the northern suburbs of Beijing. Chinese Journal of Atmospheric Sciences, 2010, 34(3): 661-668. [张宏升, 刘新建, 朱好等. 北京北郊冬季 大风过程湍流通量演变特征的分析研究. 大气科学, 2010, 34(3) : 661-668.]

[50] Wang YJ, Xu XD, Zhao TL et al. Structures of convection and turbulent kinetic energy in boundary layer over the southeastern edge of the Tibetan Plateau. Science China: Earth Sciences, 2015, 58(7) : 1198-1209.

[51] Ma YM, Wang JM, Zhang QR et al. The analysis of turbulent fluxes transfer characteristics over Nansha region. Plateau Meteorology, 1997, 16(1): 45-51. [马耀明, 王介民, 张庆荣等. 南沙海域大气湍流通量输送特征分析. 高原气象, 1997, 16(1): 45-51.]

[52] Huang R, Zhao JY, Xiao W et al. Temporal variability of radiation and energy budgets over Lake Taihu. Resources and Environment in the Yangtze Basin, 2016, 25(5): 733-742. [黄锐, 赵佳玉, 肖薇等. 太湖辐射和能量收支的时间变化 特征. 长江流域资源与环境, 2016, 25(5)：733-742.] 